\title{
RFID Based Vehicle Identification and Paid Parking System
}

\author{
Abhijith Balachandran ${ }^{\mathrm{a}}$, Saumya Ann Sam ${ }^{\mathrm{a}}$, Sweety Solanki ${ }^{\mathrm{a}}$, Varsha M. Joseph
}

\begin{abstract}
In the current scenario, parking has become a major issue as there is space, money and manual work related issues associated with it. Though the advancements in technology have brought parking facilities the way we see today, these limitations are yet to be overcome. We find it difficult to find a parking slot during peak traffic times. Payment is also difficult as it is manually done; payment is not fully automatic as it involves direct payment of cash or coin. Also, it involves payment for a fixed amount of time which might not ensure full utilization of money spent in case of leaving before the estimated time. In this project, an attempt has been made to overcome the problems encountered in the present paid parking system through RFID technology.
\end{abstract}

Keywords: cards; database; identification; payment; RFID; vehicle

\section{Introduction}

RFID or radio frequency identification is a technique that allows the identification of any item or commodity without the need of any line of sight between the transponder and reader. RFID system comprises of an antenna for scanning, a transceiver with a decoder for the interpretation of the data, and a transponder or the RFID tag that is programmed with the information. The RF radiation does two things firstly, it provides a means to communicate with the tag and secondly, it provides the RFID tag with energy needed to communicate.

The activation signal is detected from the antenna, when the RFID tag passes through the field of the scanning antenna. This signal wakes up the RFID chip, in order to transmit the information stored on its microchip, needed to be taken up by the scanning antenna. The RFID cards are acquired and database is created. The information concerning the vehicles is entered into the database. When the card is swiped, in order to check-in to the parking area the system ensures if the vehicle is registered to the database. If registered, the gate opens and the vehicle check-in time is recorded and the system displays the number of available parking slots. At the exit, the vehicle's check-out date and time are considered; check-out date and total time are subtracted from check-in date and total time. The calculated time is converted into minutes and the total fee is calculated and deducted automatically.

\section{Why RFID}

Though other identification systems like the barcode system exist, using RFID has greater advantages. Firstly, unlike barcodes several RFID tags could be read all at once by using anti-collision readers and tags without any physical contact or line of sight. RFID tag could be read, written to and rewritten to easily but once data is written to a barcode, it doesn't really support updates and any updating would be an intensive task. Data could be encrypted in case of RFID and thus is resistant to counterfeiting whereas barcodes can be duplicated thereby offering less resistance to counterfeiting. The RFID tags are also durable; reusable; could be designed to work even in harsh conditions and is capable of storing more data. [2]

\section{Literature Review}

In the "Design and Development of RFID based Automatic Car Parking System" project the researchers have fixed RFID tags to the vehicles which are read by the RFID readers which are installed at the entrance of the parking area. Each RFID tag will have the unique user password which is read by the RFID reader and is used for the validation purposes.
The proposed system can be used in various applications. It can be used in shopping malls or in an office parking area. The time factor plays a major role in such places. The digital parking management ensures that minimum time is used thus eliminating the time consumption issues. [7]

The "RFID based Digital Parking Management System" project researches have designed and tested the system under environment conditions and ideal results are accomplished. The suggested system works aptly for all the functions for which it is created. It can identify the vehicle with no or invalid tag and restrict the entry of the vehicle in the parking lot. It also facilitates the user to access the availability of parking space and will send a wait signal till the space is not free for parking. It will take only microseconds for the processing of accessing the vehicle ID. Hence, this technique is less time consuming. The suggested system results in a reliable parking solution in big cities where there is less availability of parking space. The conventional parking is replaced by this system along the sides of the streets with a multi-story parking solution. Hence the problems like less security and traffic jam during parking are reduced. [2]

\section{Problem Formulation}

This project is the extension of the existing system. Here the amount is deducted by calculating the duration of the stay of a vehicle in a parking lot and also on the basis of time spent in the parking lot. The tag will be recharged with a certain amount and this amount will be deducted after each visit. A time recording technique is used, for its realization. In the previous system, payment is not fully automatic as it involves direct payment of cash or coin. Also, it involves payment for a fixed amount of time which might not ensure full utilization of money spent in case of leaving before the estimated time. This project employs automatic card scanning instead of the swipe technique that is currently in use. Through this system the manual work is highly minimized.

Methodology

The existing technology was studied and drawbacks were noted. RFID reader of suitable range $(\mathrm{EM}-18 ; 10 \mathrm{~cm})$ with compatible cards of $125 \mathrm{kHz}$ frequency was chosen. The cards contain a certain amount and could be recharged as required. ARDUINO mega2560 was chosen to interface our components. We started with the project by interfacing RFID, LCD and stepper motor were interfaced with the ARDUINO using the software Arduino IDE version 1.6.13. The programing was done in such a way that when the cards are scanned it will check whether the id is registered in the database or not and also if sufficient balance is available or not. 
If these conditions are met then the gate opens else LCD prints the access denied message. The LCD would show the number of parking slots available at the entrance. At the exit, the vehicle's check-out date and time are considered; check-out date and total time are subtracted from check-in date and total time. The calculated time is converted into minutes and the total fee is calculated and deducted automatically.

The components of the block diagram are:

RFID READER: This project uses EM-18 RFID module which operates at a frequency of $125 \mathrm{kHz}$ and has a range of $10 \mathrm{cms}$ which is ideal for this project.

RFID CARDS: $125 \mathrm{kHz}$ cards compatible with EM-18 RFID are chosen.

ARDUINO: Arduino MEGA 2560 was used since it has higher functionality and space and is more stable in the current project.

LCD: two LCDs with $16 \times 2$ and $16 x 4$ are selected for entrance and exit respectively.

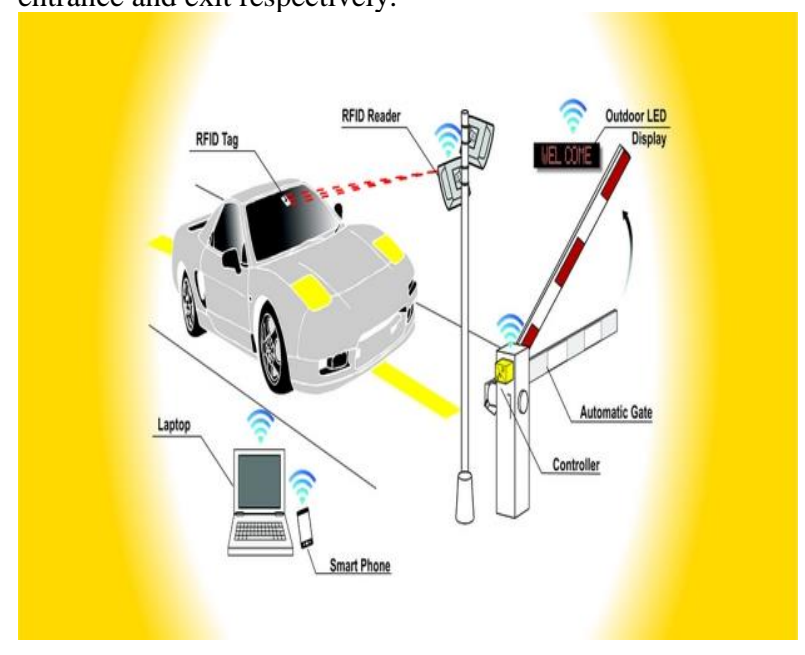

Fig. 2. Proposed System

PC: A centralized database is maintained with PC as server.

MOTOR: Stepper motor 28BYJ-48 which is 5 wire, $5 \mathrm{~V}$ DC was chosen and its motor driver was constructed with ULN2003

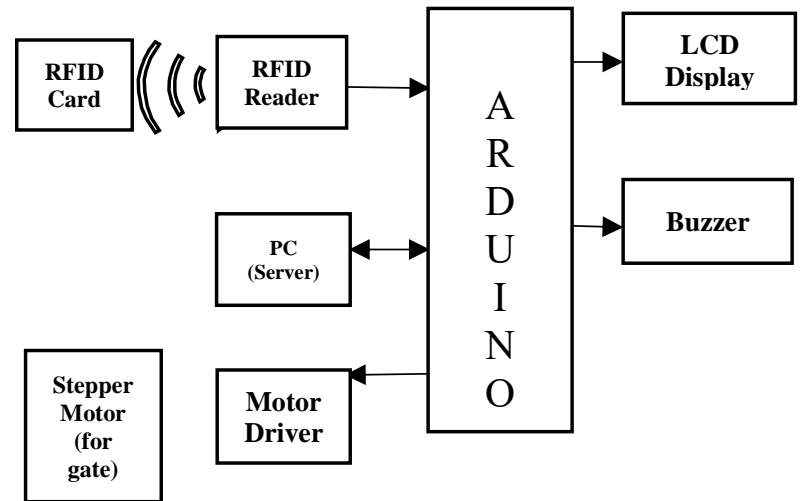

Fig. 1. Block diagram of RFID based vehicle identification and paid parking system

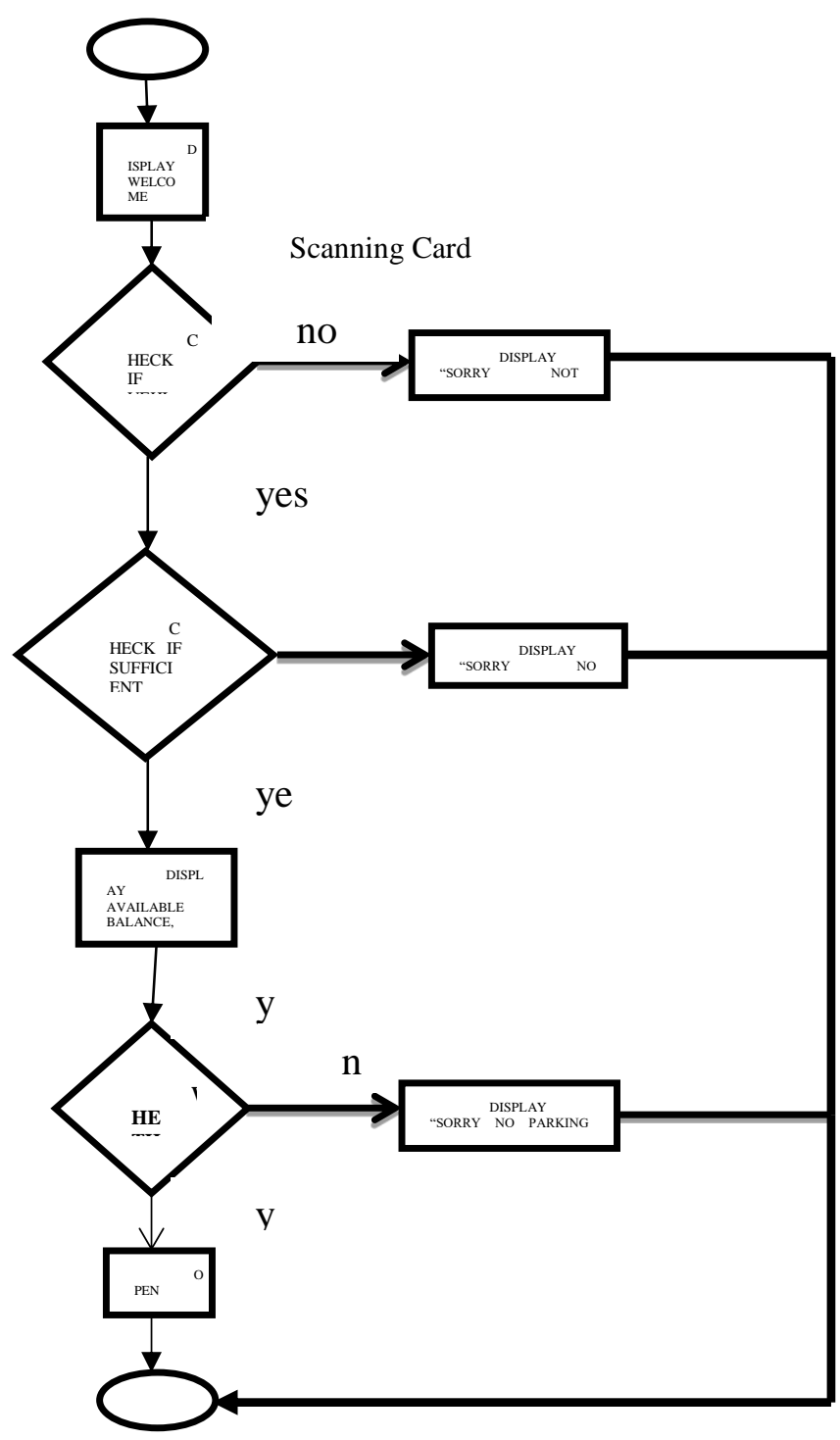




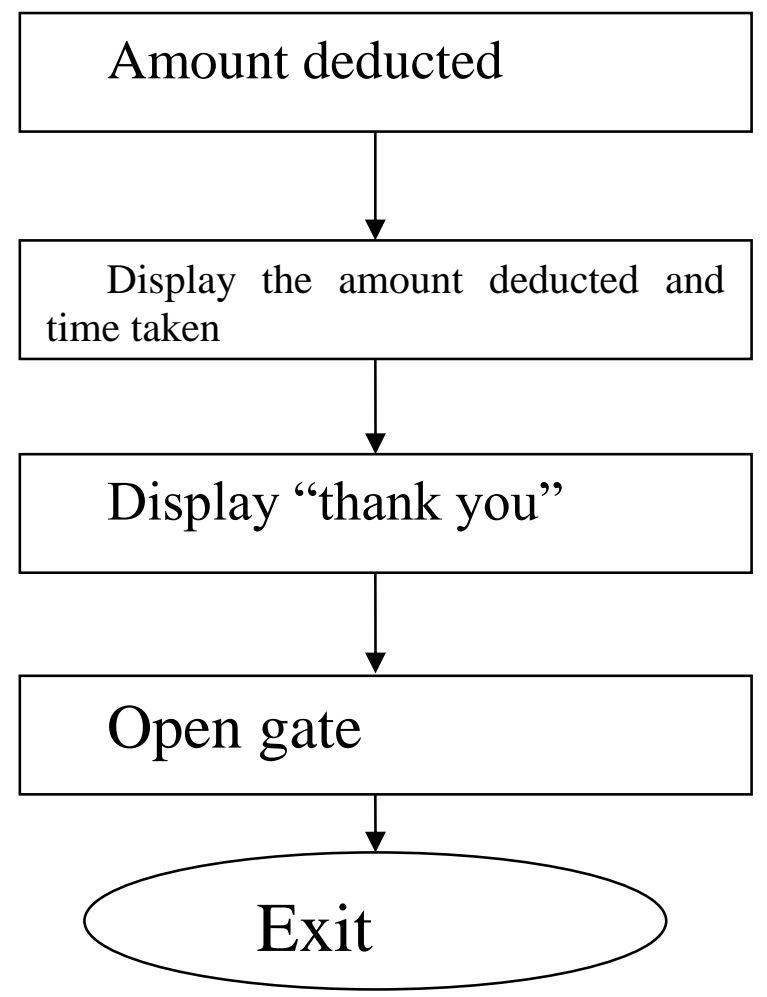

Fig.4. Flow chart - At the exit

System Requirements

\section{A. RFID Readers}

The EM-18 RFID Reader Module operates at $125 \mathrm{khz}$. It is a cost-efficient choice for your RFID based application. This RFID module contains an on-chip antenna. Reader

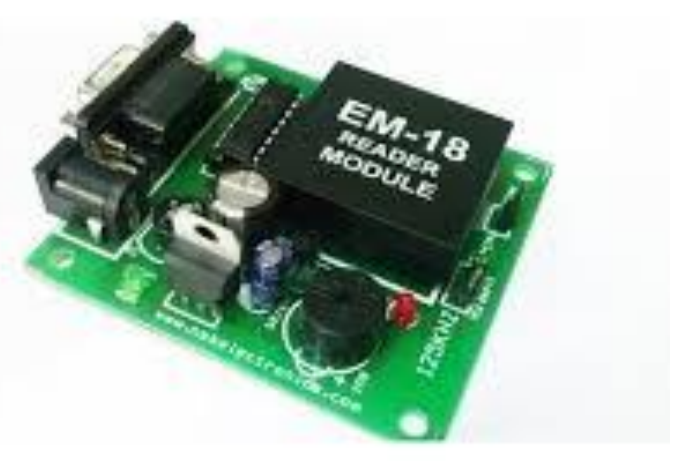

\section{RFID Cards}

The VLF RFID card that operates at $125 \mathrm{kHz}$ is used for access control related projects and functions. They are not

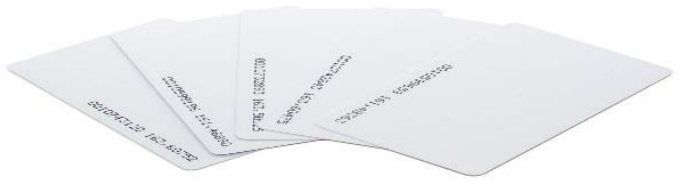

\section{Arduino Mega 2560}

This is designed for more complex projects. It is based on ATmega2560. It consists of 54 digital I/O pins, 16 analog inputs, 4 UARTs, crystal oscillator of $16 \mathrm{MHz}$. It can be powered using USB cable or with a battery. The main reason we have opted for this particular type of Arduino is because it has higher number of ports and is able to handle could be operated with a $5 \mathrm{~V}$ power supply. It consists of 9 pins, each pin separated by $2.54 \mathrm{~mm}$.Rfid EM- 18 has a range of about $10 \mathrm{~cm}$ which is ideal and perfect for this project. When the card is scanned, the card number would be shown as the output. Module could be configured for a weigand output also if required. Re-programmable and each card has a unique 32-bit ID. It is of 64-bit data stream. Two LCDs and stepper motors at the same time. It has higher functionality and space and is more stable in the current project. This Arduino is directly interfaced with the RFID reader. It could be connected to a computer with a USB cable or could be powered with an ACto-DC adapter or battery to get started.

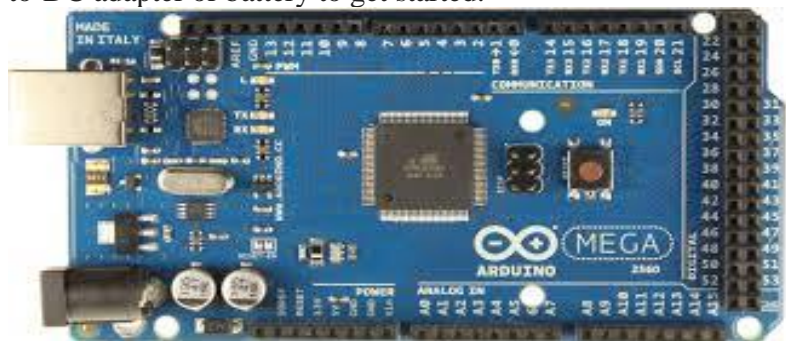

\section{Arduino 1.6.13}

Arduino 1.6.13 is an open project mainly used for programming and coding which later helps in the interaction and to interface main components such as Arduino with supportive components such as LCD, LED, RFID reader and tags etc. With the help of the above given Arduino software we have arrived at a productive and informative and highly helpful program codes which we are able to implement on our project which is the RFID BASED CAR PARKING SYSTEM. This software is adaptable for Windows 10 which is the latest update in windows. Installation and access of the software is very simple and quick. The software itself includes a beginner guide and separate information codes of various different components which can be interfaced according to users' choice.

\section{Results}

Our project is the extension of the existing system. Amount is deducted by calculating the duration of the stay of a vehicle in a parking lot. The tag will be recharged with a certain amount from which calculated amount would be deducted after each visit. A time recording technique is used, for its realization. In the previous system, payment is not fully automatic as it involves direct payment of cash or coin. Also, it involves payment for a fixed amount of time which might not 
ensure full utilization of money spent in case of leaving before the estimated time. This project employs automatic card scanning instead of the swipe technique that is currently in use. The congestion in the parking area could be reduced and thereby time could be saved. Through this system the manual work is highly minimized.

\section{Impact}

The congestion in the parking area could be reduced thereby saving time. Since this project ensures easy payment based on the duration spent and not on any predetermined amount for particular time as in current system, the visitors can leave at their convenient time without any money wastage, this is one keynote in this project.

\section{Application}

This project could be used for banks, industries, offices and other establishments.

\section{Future scope}

To reduce vehicle theft and to monitor reserved parking spaces, security cameras could be installed.

In order to prevent anti socialist activities and parked car explosions, weight sensors and bomb detection tools could be installed in the parking areas. Parking Levels like VIP or General parking can also be implemented. GSM module could be incorporated to send the parking lot subscribers notifications via SMS, informing them about the availability of parking slots.

\section{Conclusion}

This RFID enabled paid parking system that uses RFID readers and cards with a centralized database system enables fast and economical parking.

The technology ensures less waiting time at the entry and exit barriers of the parking lot and is a more economical approach as compared to the regular barcode scanned or manned parking system.

\section{Acknowledgements}

We owe the completion of our project to God Almighty. We express our sincere thanks to our professors Mr.Rajeev Ranjan and Mr. Praveen Sanga, the Guides of our project, for their guidance and support. We also reveal our heartfelt gratitude to Mrs. Anasuya Patil and Mr. Shyam Sundar Purohit for their assistance provided without which this project would not have been completed. We are also grateful to our Institution, Waljat College of Applied Sciences and the faculty members. We also extend our heartfelt thanks to our familes and well-wishers without whom our project would not have been completed.

\section{References}

[1]2017.[Online].Available: https://www.ijircce.om/upload/2016/february/187_RFID.pdf.[Accesse d:05-May-2017].

[2][Online].Available:

http://ijafrse.org/Volume1/vivuti/27.pdf. Accessed: Jan 2,2017
[3]2017.[Online].Available:

https://www.researchgate.net/publication/295979433 Rfid_Based_Aut omatic Parking System.[Accessed:05-May-2017].

[4]"Smart Parking Applications Using RFID Technology-IEEE Xplore Document",Ieeexplore.ieee.org,2017.[Online].Available: http://ieeexplore.ieee.org/document/4368108/.[Accessed: 05-May2017

[5]2017.[Online].Available:

http://www.ijettjournal.org/2016/volume-32/number-4/IJETTV32P238.pdf.[Accessed: 05-May-2017].

[6]"Redirecting", Google.com, 2017 . [Online]. Available:

https://www.google.com/url?q=https $\% 3 \mathrm{~A} \% 2 \mathrm{~F} \% 2 \mathrm{Fwww}$. researchgate. net\%2Fpublication\%2F4285055 Smart_Parking_Applications_Using RFID Technology\&sa=D\&sntz=1\&usg=AFQjCNGv24t YLL7LRs kSdSITukHqlPf2A. [Accessed: 05-May-2017].

[7]2017. [Online]. Available:

https://www.researchgate.net/profile/Ekta Soni3/publication/2773293 91 Design And Development Of RFID Based Automated Car Pa rking_SYSTEM/links/5777790b408ae4645d60ff3e9.pdf?origin=publi cation_detail. [Accessed: 05-May_2017]. 